\title{
The impact of using different lean manufacturing tools on waste reduction
}

\author{
Leksic, l. ${ }^{a,}{ }^{,}$, Stefanic, N. ${ }^{a}$, Veza, I. ${ }^{b}$ \\ aniversity of Zagreb, Faculty of Mechanical Engineering and Naval Architecture, Zagreb, Croatia \\ ${ }^{b}$ University of Split, Faculty of Electrical Engineering, Mechanical Engineering and Naval Architecture, Split, Croatia
}

\section{A B S T R A C T}

Lean and green production was introduced to the western manufacturing industry nearly thirty years ago. The essence of the new business model was to eliminate waste through lean tools according to Taiichi Ohno's eight categories of waste. Many companies became more competitive with waste reduction techniques but some of them faced, and still are facing failures. Such failures are closely related with misapplication of lean and green tools, and its sequential order of implementation. In order to define most powerful lean tools for reduction of certain types of waste, a study was made among lean companies. The concept of a study was to define best lean toolbox for reduction of each category of waste and to determine right sequential order of lean tools implementation. Stepwise multiple regression model revealed that Total Productive Maintenance, Poka-Yoke, Kaizen, 5S, Kanban, Six Big Losses, Heijunka, Takt Time, Andon, OEE, SMED, and KPIs are best waste management techniques. Nevertheless, it has been demonstrated that 5S, Kaizen, Kanban, Poka-Yoke and TPM are highly recommended for start of every lean manufacturing initiative.
\end{abstract}

\author{
ARTICLE INFO \\ Keywords: \\ Green production; \\ Lean manufacturing; \\ Lean tools; \\ Waste reduction; \\ Waste management; \\ Waste reduction techniques \\ ${ }^{*}$ Corresponding author: \\ ileksic@gmx.com \\ (Leksic, I.) \\ Article history: \\ Received 17 September 2019 \\ Revised 4 March 2020 \\ Accepted 17 March 2020
}

\section{References}

[1] Marodin, G.A., Saurin, T.A. (2013). Implementing lean production systems: Research areas and opportunities for future studies, International Journal of Production Research, Vol. 51, No. 22, 6663-6680, doi: 10.1080/00207543. 2013.826831.

[2] Baker, P. (2002). "Why is lean so far off? If lean manufacturing has been around for decades, why haven't more manufacturers got further with it?", Works Management -London then Horton Kirby then Swanley-, Vol. 55, No. 10, 26-29.

[3] Mohanty, R., Yadav, O., Jain, R. (2007). Implementation of lean manufacturing principles in auto industry, Vilakshan-XIMB Journal of Management, Vol. 1, No. 1, 1-32.

[4] Taylor, A., Taylor, M., McSweeney, A. (2013). Towards greater understanding of success and survival of lean systems, International Journal of Production Research, Vol. 51, No. 22, 6607-6630, doi: 10.1080/00207543.2013. 825382.

[5] Yadav, O.P., Nepal, B., Goel, P.S., Jain R., Mohanty, R.P. (2010). Insights and learnings from lean manufacturing implementation practices, International Journal of Services and Operations Management, Vol. 6, No. 4, 398-422, doi: 10.1504/IJSOM.2010.032916.

[6] Liker, J.K., Meier, D.P. (2006). The Toyota way fieldbook: A practical guide for implementing Toyota's 4Ps, McGrawHill, New York, USA.

[7] Conti, T. (2010). The dynamics of value generation and their dependence on an organisation's internal and external value system, Total Quality Management \& Business Excellence, Vol. 21, No. 9, 885-901, doi: 10.1080/ 14783363.2010 .487701$.

[8] Mostafa, S., Dumrak, J., Soltan, H. (2013). A framework for lean manufacturing implementation, Production \& Manufacturing Research, Vol. 1, No. 1, 44-64, doi: 10.1080/21693277.2013.862159. 
[9] Pavnaskar, S.J., Gershenson, J.K., Jambekar, A.B. (2003). Classification scheme for lean manufacturing tools, International Journal of Production Research, Vol. 41, No. 13, 3075-3090, doi: 10.1080/0020754021000049817.

[10] Koblasa, F., Šírová, E., Králíková, R. (2019). The use of process thinking in the industrial practice - Preliminary survey, Tehnički Vjesnik - Technical Gazette, Vol. 26, No. 3, 786-792, doi: 10.17559/TV-20150617135306.

[11] Sim, K.L., Rogers, J.W. (2009). Implementing lean production systems: Barriers to change, Management Research News, Vol. 32, No. 1, 37-49, doi: 10.1108/01409170910922014.

[12] Fullerton, R.R., Wempe, W.F. (2009). Lean manufacturing, non-financial performance measures, and financial performance, International Journal of Operations \& Production Management, Vol. 29, No. 3, 214-240, doi: 10.1108 $\not 01443570910938970$.

[13] Henderson, B.A., Larco, J.L. (2003). Lean transformation: How to change your business into a lean enterprise, Oaklea Press, Virginia, USA.

[14] Liker, J.K. (2004). The Toyota way: 14 management principles from the world's greatest manufacturer, McGrawHill, New York, USA.

[15] Wiengarten, F., Fynes, B., Pagell, M., de Búrca, S. (2011). Exploring the impact of national culture on investments in manufacturing practices and performance: An empirical multi-country study, International Journal of Operations \& Production Management, Vol. 31, No. 5, 554-578, doi: 10.1108/01443571111126328.

[16] Achanga, P., Shehab, E., Roy, R., Nelder, G. (2006). Critical success factors for lean implementation within SMEs, Journal of Manufacturing Technology Management, Vol. 17, No. 4, 460-471, doi: 10.1108/17410380610662889.

[17] Nguyen, D.M. (2015). A new application model of lean management in small and medium sized enterprises, International Journal of Simulation Modelling, Vol. 14, No. 2, 289-298, doi: 10.2507/IISIMM14(2)9.304.

[18] Ohno, T. (1988). Toyota production system - Beyond large-scale production, 1st edition, Productivity Press, New York, USA, doi: $10.4324 / 9780429273018$.

[19] Anand, G., Kodali, R. (2010). Analysis of lean manufacturing frameworks, Journal of Advanced Manufacturing Systems, Vol. 9, No. 1, 1-30, doi: 10.1142/S0219686710001776.

[20] Powell, D., Riezebos, J., Strandhagen, J.O. (2012). Lean production and ERP systems in small-and medium-sized enterprises: ERP support for pull production, International Journal of Production Research, Vol. 51, No. 2, 395409, doi: $10.1080 / 00207543.2011 .645954$.

[21] Netland, T.H. (2015). Critical success factors for implementing lean production: The effect of contingencies, International Journal of Production Research, Vol. 54, No. 8, 2433-2448, doi: 10.1080/00207543.2015.1096976.

[22] Garcia-Sabater, J.J., Marin-Garcia, J.A. (2011). Can we still talk about continuous improvement? Rethinking enablers and inhibitors for successful implementation, International Journal of Technology Management, Vol. 55, No. 1-2, 28-42, doi: 10.1504/IJTM.2011.041678.

[23] Emiliani, B. (2008). Practical lean leadership - A strategic leadership guide for executives, Center for Lean Business Management, Wethersfield, USA.

[24] Kolberg, D., Zühlke, D. (2015). Lean automation enabled by Industry 4.0 technologies, IFAC-PapersOnLine, Vol. 48, No. 3, 1870-1875, doi: 10.1016/i.ifacol.2015.06.359.

[25] Narasimhan, R., Swink, M., Kim, S.W. (2006). Disentangling leanness and agility: An empirical investigation, Journal of Operations Management, Vol. 24, No. 5, 440-457, doi: 10.1016/j.jom.2005.11.011.

[26] Čiarnienè, R., Vienažindienè, M. (2015). An empirical study of lean concept manifestation, Procedia - Social and Behavioural Sciences, Vol. 207, 225-233, doi: 10.1016/i.sbspro.2015.10.091.

[27] Browning, T.R., Heath, R.D. (2009). Reconceptualising the effects of lean on production costs with evidence from the F-22 program, Journal of Operations Management, Vol. 27, No. 1, 23-24, doi: 10.1016/i.jom.2008.03.009.

[28] Yadav, O.P., Nepal, B.P., Rahaman, M.M., Lal, V. (2017). Lean implementation and organizational transfor-mation: A literature review, Engineering Management Journal, Vol. 29, No. 1, 2-16, doi: 10.1080/10429247.2016.126 $\underline{3914 .}$

[29] Bhasin, S., Burcher, P. (2006). Lean viewed as a philosophy, Journal of Manufacturing Technology Management, Vol. 17, No. 1, 56-72, doi: 10.1108/17410380610639506.

[30] Vorkapić, M., Radovanović, F., Ćoćkalo, D., Đorđević, D. (2017). Applicability of the lean concept to the management of small-scale manufacturing enterprises in Serbia, Tehnički Vjesnik - Technical Gazette, Vol. 24, No. 6, 1929-1934, doi: 10.17559/TV-20150807194942.

[31] Karlsson, C., Åhlström, P. (1996). Assessing changes towards lean production, International Journal of Operations \& Production Management, Vol. 16, No. 2, 24-41, doi: 10.1108/01443579610109820.

[32] Kadarova, J., Demecko, M. (2015). New approaches in lean management, Procedia Economics and Finance, Vol. 39, 11-16, doi: 10.1016/S2212-5671(16)30234-9.

[33] Arunagiri, P., Gnanavelbabu, A. (2014). Identification of high impact lean production tools in automobile industries using weighted average method, Procedia Engineering, Vol. 97, 2072-2080, doi: 10.1016/i.proeng.2014.12. $\underline{450}$.

[34] Holtskog, H. (2013). Continuous improvement beyond the lean understanding, Procedia CIRP, Vol. 7, 575-579, doi: 10.1016/j.procir.2013.06.035.

[35] Antosz, K., Stadnicka, D. (2017). Lean philosophy implementation in SMEs - Study results, Procedia Engineering, Vol. 182, 25-32, doi: 10.1016/j.proeng.2017.03.107.

[36] Vilkas, M., Koreckaja, I., Katiliūtė, E., Bagdonienè, D. (2015). Adoption of lean production: Preliminary evidence from Lithuania, Procedia - Social and Behavioural Sciences, Vol. 213, 884-889, doi: 10.1016/j.sbspro.2015. $\underline{11.500}$. 


\title{
APEM
}

\section{Vpliv uporabe različnih vitkih proizvodnih orodij na zmanjševanje odpadkov}

\author{
Leksic, l..$^{a,}$, Stefanic, N. ${ }^{a}$, Veza, I. ${ }^{b}$ \\ ${ }^{a}$ University of Zagreb, Faculty of Mechanical Engineering and Naval Architecture, Zagreb, Croatia \\ ${ }^{b}$ University of Split, Faculty of Electrical Engineering, Mechanical Engineering and Naval Architecture, Split, Croatia
}

\section{POVZETEK}

Vitka in zelena proizvodnja je bila zahodni proizvodni industriji predstavljena pred skoraj tridesetimi leti. Bistvo novega poslovnega modela je bilo odstranjevanje odpadkov s pomočjo vitkih orodij v skladu z osmimi kategorijami odpadkov Taiichija Ohna. Številna podjetja so postala bolj konkurenčna s tehnikami zmanjševanja odpadkov, vendar pa so se nekatera izmed njih soočala in se še vedno soočajo z napakami. Napake so tesno povezane z nepravilno uporabo vitkih in zelenih orodij ter $\mathrm{z}$ vrstnim redom izvajanja teh orodij. Da bi opredelili najprimernejša vitka orodja za zmanjševanje posameznih vrst odpadkov, je bila narejena študija, ki je vključevala več vitkih podjetij. Koncept študije je bil določiti najboljši nabor vitkih orodij za zmanjšanje posamezne kategorije odpadkov in določiti pravi vrstni red izvajanja vitkih orodij. Model postopne večkratne regresije je razkril, da so Total Productive Maintenance, Poka-Yoke, Kaizen, 5S, Kanban, Six Big Loses, Heijunka, Takt Time, Andon, OEE, SMED in KPI najboljše tehnike ravnanja z odpadki. Dodatno je bilo dokazano, da so 5S, Kaizen, Kanban, Poka-Yoke in TPM zelo priporočljive začetne tehnike vzpostavljanja vitke proizvodne.

\section{PODATKI O ČLANKU}

Ključne besede:

Zelena proizvodnja

Vitka proizvodnja

Vitko orodje

Zmanjševanje odpadkov

Ravnanje z odpadki

Tehnike zmanjševanja odpadkov

*Kontaktna oseba:

ileksic@gmx.com

(Leksic, I.)

Zgodovina članka:

Prejet 17. septembra 2019

Popravljen 4. marca 2020

Sprejet 17. marca 2020 